\title{
On the Characterization of the Geometrical Collection Efficiency of Modern EDS Systems
}

\author{
Ralf Terborg $^{1}$, Vasile-Dan Hodoroaba ${ }^{2}$, Meiken Falke $^{1}$ and Andi Käppel ${ }^{1}$ \\ ${ }^{1}$ Bruker Nano GmbH, Am Studio 2D, 12489 Berlin, Germany \\ ${ }^{2}$ BAM Federal Institute for Materials Research and Testing, 12200 Berlin, Germany
}

To compare the performance of different energy dispersive X-ray spectrometers (EDS), it is important to define characteristic spectrometer parameters. The ISO 15632 standard defines parameters like energy resolution as FWHM for the $\mathrm{K} \alpha$ lines of carbon, fluorine and manganese. The quantum efficiency, which is the ratio of the detected photons divided by the number of incoming photons for different energies, is another significant spectrometer property. It is important for the light element and low energy line detection sensitivity as well as for higher photon energies above $10 \mathrm{keV}$. A striking EDS feature, provided and marketed by many manufactures, is the active area of the detector, although actually, the solid angle available for photon collection is the more relevant geometrical parameter. It is defined as: $\Omega=A / r^{2}$ with $A$ being the active area of a spherical detector and $r$ being the distance between the point of the radiation origin and the center of the surface of the active detector chip. A more accurate calculation should be used for large flat detector areas [1]. One should note that the solid angle $\Omega$ is not an intrinsic spectrometer property. It can only be defined for a specific detector in combination with a specific system (e.g. SEM, EPMA or TEM). Thus, the minimal possible distance $r$ is determined by the particular geometry e.g. a possible interference with the pole piece or other detectors/components in the chamber of a microscope. New EDS technologies use e.g. integrated SDD chips or inclined chips in thinner detector fingers which can be placed closer to the sample with the final result of larger real solid angles. Therefore, the knowledge of the real solid angle is one of the crucial parameters of an EDS microscope combination. A straightforward way to estimate the real solid angle is to simply determine $A$ and $r$. If respective data are not provided by the manufacturer, this approach can be difficult.

For TEM, the solid angle can be estimated from the ratio of the measured to the theoretical X-ray net count number in a specific element line using a sample of known thickness (e.g. $\mathrm{NiO}_{\mathrm{x}}$ on a Mo grid) at well-defined acquisition conditions [2]. Parameters such as the detector take-off angle, quantum efficiency, collimator design, background causing sample holder and microscope aperture materials, geometry and also the electron beam quality influence the experimental result though and need to be known and under control. If this is the case, the approach can deliver results quite close to the real geometric solid angle. Otherwise it can be used as a performance parameter to compare EDSmicroscope combinations as suggested by manufacturers of standard samples.

A similar approach to find the real solid angle or a parameter which makes solid angles comparable for SEM/EDS systems is to acquire an X-ray spectrum under defined conditions, e.g. a spectrum of a pure $\mathrm{Cu}$ bulk sample at $20 \mathrm{keV}$ accelerating voltage at known beam current and measure the number of counts in the $\mathrm{Cu}-\mathrm{K} \alpha+\mathrm{K} \beta$ peaks as proposed recently by Schamber [3]. Additionally to the beam current the input count rate must be known. Using high energy lines reduces the influence of absorption effects due to a different detector take-off angle at different microscopes, which normally varies between $30^{\circ}$ and $40^{\circ}$, or sample surface morphology or contamination. On the other hand some SEMs don't have the possibility to measure the beam current or don't have a well calibrated ampere meter. Furthermore, the quantum efficiency, which is different between a detector with different windows or a windowless 
detector or detectors with different contact layers must be known. It is also important to take the dead time into account. With a significant dead time not the output count rate but the input count rate must be used.

An approach we suggest to determine the real detector-sample distance for inclined EDS ports as typical for SEM-EDS is, to measure the count rate in a defined energy region (e.g. for $\mathrm{Cu}$ in the range [7.5 keV, $9.5 \mathrm{keV}]$ ) for various detector positions by retracting the detector in known steps without altering the take-off angle and keeping the electron optics setup unchanged. This can be carried out even without knowing the beam current - if stable! - or the detector efficiency. The count rate $I$ should be proportional to $1 / r^{2}$ and therefore, $1 / \sqrt{ } I$ vs. $r$ should be a straight line through the origin. This can be used to determine the absolute distance, see Fig 1. For the active area A the nominal value can be used. Alternatively (if possible), measurements with apertures of known area placed onto the front of the EDS in a fixed measurement position can be used [4]. In the latter case the exact position of the active chip must be known (from manufacturer). After measuring without apertures and extrapolating the results obtained with the apertures, the real active area can be determined accurately.

\section{References:}

[1] N J Zaluzec, Microsc. Microanal., 15 (2009) p. 93.

[2] R F Egerton, S C Cheng, Ultramicroscopy, 55 (1994), p. 43.

[3] F Schamber, proposal at the 19th meeting of ISO/TC202, Boulder, CO, USA, 2013.

[4] M Procop, Microsc. Microanal, 10 (2004), p. 481.

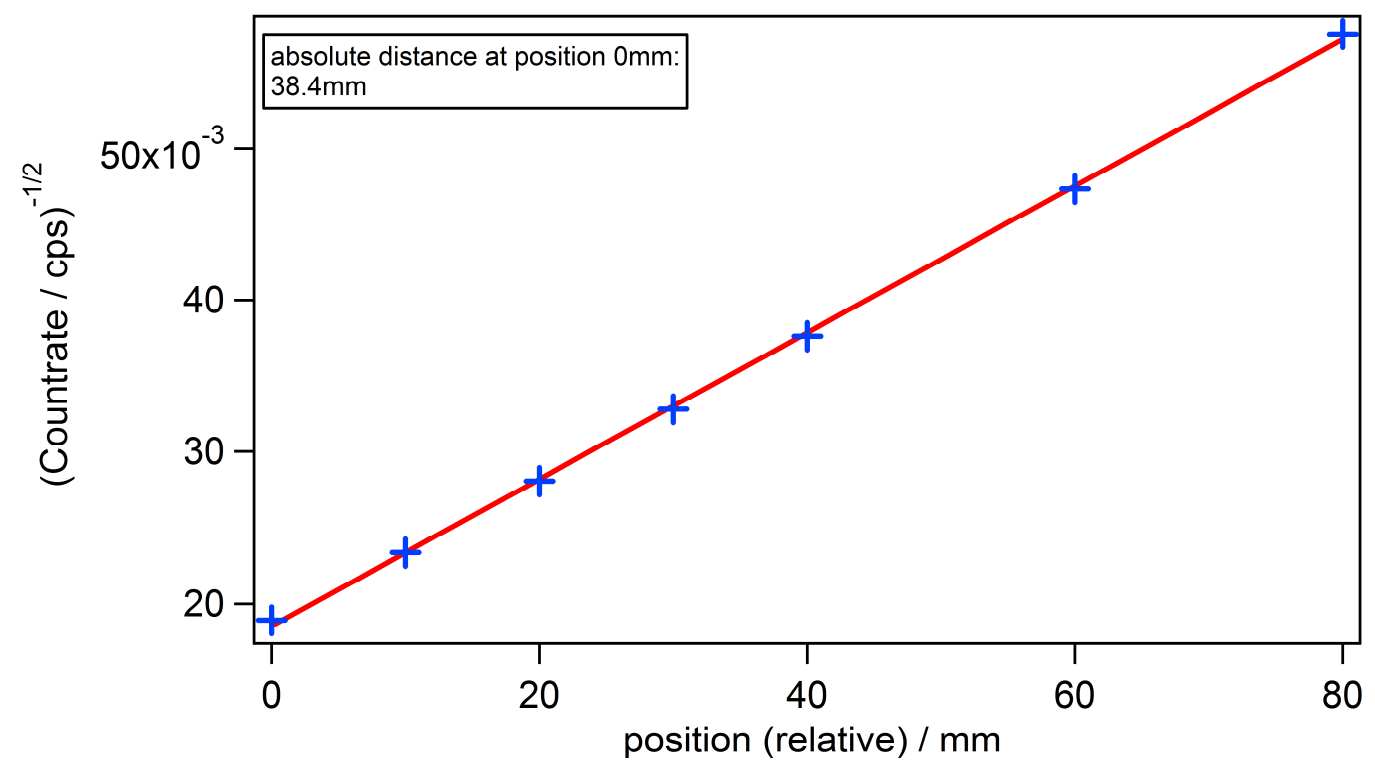

Fig. 1. Count rate parameter (expressed as $\mathrm{cps}^{-1 / 2}$ ) in dependence on the relative position of the EDS for the calculation of the absolute distance from the radiation origin to the detector chip. 